\begin{tabular}{l|l} 
REVISTA & $\begin{array}{l}\text { Revista Educación } \\
\text { ISSN: 0379-7082 } \\
\text { ISSN: 2215-2644 } \\
\text { revedu@gmail.com } \\
\text { Universidad de Costa Rica } \\
\text { Costa Rica }\end{array}$
\end{tabular}

\title{
La función formativa de la evaluación en el trabajo escolar cotidiano
}

\author{
Segura Castillo, Mario Alberto \\ La función formativa de la evaluación en el trabajo escolar cotidiano \\ Revista Educación, vol. 42, núm. 1, 2018 \\ Universidad de Costa Rica, Costa Rica \\ Disponible en: http://www.redalyc.org/articulo.oa?id=44051918010 \\ DOI: https://dx.doi.org/10.15517/revedu.v42i1.22743
}

Esta obra está bajo una Licencia Creative Commons Atribución-NoComercial-SinDerivar 3.0 Internacional 


\section{La función formativa de la evaluación en el trabajo escolar cotidiano}

\section{The Training Function of Evaluation in Everyday School Work}

Mario Alberto Segura Castillo [1]

Universidad de Costa Rica, Costa Rica

DOI: https://dx.doi.org/10.15517/revedu.v42i1.22743

mario.segura@ucr.ac.cr

Redalyc: http://www.redalyc.org/articulo.oa?id=44051918010

Recepción: 06 Enero 2016

Aprobación: 13 Julio 2017

\section{RESUMEN:}

La función formativa, cuyo propósito es regular el proceso educativo en el aula, es decir, el trabajo escolar cotidiano, el cual se integra a la intervención didáctica ya que está centrada en los procesos más que en los resultados de aprendizaje, por lo que la realimentación-proalimentación que se obtiene es de carácter cualitativo. Ello permite detectar las barreras de aprendizaje para el desarrollo de la mediación pedagógica del estudiantado. Se pretende en la presente investigación realizar una reflexión con la opinión de docentes del III Ciclo y Educación Diversificada del sistema educativo costarricense, con la finalidad de enriquecer la práctica evaluativa, con aspectos que hasta hoy no han sido importantes, tal es el caso de la función formativa que conlleva tomar en cuenta la diversidad desde procesos como la realimentación, proalimentación, la sostenibilidad de la evaluación y las barreras para el aprendizaje. Se concluye que el personal docente observado y posteriormente entrevistado aún no logra tener claridad sobre la forma en que se califica el componente de trabajo cotidiano.

PALABRAS ClAVE: evaluación formativa, realimentación, proalimentación, evaluación entre iguales.

\section{Abstract:}

The formative function, which purpose is to regulate the educational process in the classroom, meaning the daily school work, which is integrated to the didactic intervention since it is focused on the processes rather than on the learning outcomes, for what the feedback-that is obtained is of qualitative character, allows to detect the learning barriers for the development of the pedagogical mediation of the student body. The aim of this research is to reflect on the opinion of the teachers of the III Cycle and Diversified Education of the Costa Rican Educational System, with the purpose of enriching the evaluation practice with aspects that have not been important to this day such is the case of the formative function that entails taking into account diversity from processes such as feedback, feedback, sustainability of assessment and barriers to learning. It is concluded that the teaching staff observed and subsequently interviewed still cannot be clear how the daily work component is qualified.

KEYWORDS: formative evaluation, feedback, feedforward, peer review.

\section{INTRODUCCIÓN}

La evaluación de los aprendizajes debe ser contextualizada en los procesos de enseñanza y aprendizaje, además, utilizar la función formativa, dentro de ambientes significativos del estudiantado, por medio de situaciones problema. Según, Condemarín y Medina (2000), las bases teóricas de la evaluación auténtica se derivan de la evaluación formativa. Sin embargo, amplía su proyección, ya no estando en un momento específico, sino como una herramienta en todo momento, la cual realimenta - proalimenta no solo al estudiantado, sino al personal docente. Otras teorías consideradas en la construcción de este modelo son la teoría del esquema

\section{NotAS DE AUTOR}

[1] Doctor en Educación con Especialidad en Mediación Pedagógica de la Universidad La Salle, Costa Rica. Magister en Evaluación Educativa, Universidad de Costa Rica; Licenciado en Administración Educativa de la Universidad de Costa Rica. Magister en Educación en Democracia y Valores en Iberoamérica, Universidad de Barcelona (2002 - 2003). Profesor universitario de la Universidad de Costa Rica y la Universidad Nacional. Miembro de la Comisión Institucional de Educación en Formación en Valores del Ministerio de Educación Pública. Asesor Nacional de Educación Especial Ministerio de Educación Pública Profesor interino Escuela de Orientación y Educación Especial, UCR. 
(cognitivo), la perspectiva ecológica/socio-cognitiva complementada con la perspectiva etnográfica, el socio constructivismo y la práctica pedagógica crítica-reflexiva.

Según Salinas, Rodríguez y Cuervo (2013), las anteriores corrientes epistemológicas no entran en contradicción, debido a tratarse de un paradigma interpretativo o cualitativo que lo que pretende es, precisamente:

Centrar sus esfuerzos en la comprensión de los significados de las acciones humanas, y esto se verá reflejado en nuestro caso en la evaluación. Así, la finalidad de la investigación evaluativa, bajo [sic] esta mirada, es la comprensión de los fenómenos educativos a través del análisis profundo de las percepciones e interpretaciones de los sujetos intervinientes en las diversas situaciones. (p. 3)

De acuerdo con lo anterior, la finalidad del trabajo escolar cotidiano es, precisamente, centrarse en la percepción del personal docente, comprender e interpretar la aplicación de la función formativa mediante procesos de realimentación y proalimentación. Este último, conlleva optimar el progreso en cuanto a los procesos de enseñanza y aprendizaje de la población estudiantil en el presente, igualmente a mantener una visión positiva hacia el futuro, incluso se puede hacer una valoración, con la coevaluación, la evaluación entre iguales y la autoevaluación. De acuerdo con Rodríguez e Ibarra (2011), proporciona que:

El profesor, como un miembro más de la comunidad de práctica del estudiante, facilita el diálogo con el estudiante para que éste utilice toda la información y decida cómo regular su aprendizaje hasta alcanzar el nivel de referencia fijada para una actuación o producto presente o futuro. (p. 6)

La proalimentación en el salón de clases se evidencia cuando el personal docente solicita un trabajo reiteradamente para que en cada entrega sus estudiantes tengan múltiples oportunidades de irlo mejorando, así como en el futuro aplicar lo aprendido ante otras situaciones.

Por tanto, Casanova (1999) visualiza la función formativa como proceso continuo, debido a que accede, durante el desarrollo del proceso de aprendizaje, a cambios metodológicos, adecua el proceso de enseñanza; considera que esta debe plasmarse en facilitar estrategias de aprendizaje a la población estudiantil, con el uso de otras técnicas metodológicas que faciliten interactuar activamente con el personal docente, entonces, realimentar el proceso durante el desempeño en las actividades que se efectúan a nivel áulico.

Consecuentemente, el trabajo escolar cotidiano es, precisamente, todas las actividades donde el cuerpo docente tiene la oportunidad de representar, de múltiples formas, el proceso de enseñanza y el estudiantado tenga la oportunidad de expresarse de diversas maneras, utilizar la diversidad de medios, técnicas y estrategias en el proceso de aprendizaje, a la vez propiciar un ambiente motivacional, donde el aprendizaje va de la mano con la función formativa de la evaluación de los aprendizajes.

En el Reglamento de Evaluación de los Aprendizajes de Costa Rica (Ministerio de Educación Pública [MEP], 2009), se conceptúa:

Trabajo cotidiano [como] todas las actividades educativas que realiza el alumno con la guía del docente. Este trabajo se observa en forma continua, durante el desarrollo de las lecciones, como parte del proceso de aprendizaje y no como producto. Para su calificación se debe utilizar la información recopilada con las escalas de calificación y otros instrumentos técnicamente elaborados. (p. 20)

Desde ese punto de vista, en el proceso de mediación pedagógica para la calificación del "trabajo escolar cotidiano" (TEC), permite recolectar evidencias del aprendizaje del estudiantado por medio de la técnica de la observación; asimismo, requiere la aplicación de innovaciones curriculares, por ser el espacio propicio donde el personal docente aplica diferentes técnicas y estrategias didácticas, lo cual incentiva a la población estudiantil a la participación activa del proceso de aprendizaje, de acuerdo con la diversidad del estudiantado, entre esta: población migrante, privada económicamente, con alta dotación, con necesidades educativas, con discapacidad y otros, que tradicionalmente ha sido invisibilizada y excluida. 
La función formativa de la evaluación de los aprendizajes conlleva tomar en cuenta los apoyos pedagógicos a la diversidad en el salón de clases. Precisamente, se parte en entender los procesos de aprendizaje junto a la evaluación, con el propósito de que el personal docente utilice múltiples técnicas y estrategias, con el fin de tomar en cuenta diversos estilos y ritmos de aprendizaje, el contexto, procedencia, la condición socioeconómica de la población estudiantil, en la eliminación de todo tipo de barreras.

De acuerdo con lo anterior, se plantea el siguiente problema de investigación:

¿Cuál es la relación entre la función formativa de la evaluación de los aprendizajes con los procedimientos que realiza el personal docente en la calificación del trabajo cotidiano en las diversas asignaturas en el III de la Educación General Básica y la Educación Diversificada del Sistema Educativo Diurno Público de Costa Rica, direcciones regionales de educación de San José Norte, Central, Cartago, Heredia, San Carlos y Coto?

\section{Marco conceptual}

A continuación, se presenta el referencial teórico de la investigación. Se parte de los aportes de la pedagogía crítica y el constructivismo a la función formativa de la evaluación; de la importancia de la función formativa en la mediación pedagógica; además cuál es la relación con los procesos fundamentales de la función formativa como lo son la realimentación y proalimentación, y por último, se define el trabajo escolar cotidiano, desde el Ministerio de Educación Pública de Costa Rica.

De acuerdo con Salinas y Rodríguez (2013), la pedagogía crítica pretende "no solo develar las fuerzas opresivas de la escuela, sino también reconocer el potencial de formación que en ella existe, de manera que los alumnos puedan potenciar su capacidad de transformar la realidad. Y este es un trabajo que no se hace al margen del profesor" (p. 2).

Según esta perspectiva se presenta la mediación pedagógica desde la reflexión, acción y transformación de la realidad, donde mediante una metodología participativa, el personal docente y el estudiantado realizan un debate, análisis y diálogo del contexto, con el fin de desentrañar todas aquellas prácticas que atentan con la libertad humana, con la finalidad del empoderamiento de la comunidad educativa.

Entonces, la evaluación de los aprendizajes desde el paradigma crítico, con base en Salinas y Rodríguez (2013), propone:

Una revisión crítica y colectiva sobre el trabajo desarrollado. Crítica, porque supone observar y valorar, desde los objetivos, los intereses y las necesidades de la acción educativa, la utilidad de las actividades y la correspondencia con los principios del proceso emprendido; y colectiva, porque, en coherencia con los principios de la evaluación crítica, todos los sujetos son participantes deliberativos, críticos y propositivos. (p. 4)

En suma, desde una perspectiva de la pedagogía crítica, la evaluación de los aprendizajes deja de cumplir una función eminentemente instrumental, que se le había designado al visualizarla como simple medición de conocimientos, a otra donde lo importante es que se dialogue democráticamente acerca de los procesos de aprendizajes para que el estudiantado tenga una participación plena en la calificación del trabajo escolar cotidiano y demás componentes, desde el propósito formativo de la evaluación de los aprendizajes.

El TEC condiciona la función docente, cuando se convierte en la persona facilitadora del proceso de aprendizaje, por tanto, utiliza la técnica de la observación con el fin de evidenciar aciertos y errores para encauzar el logro de los aprendizajes de la población estudiantil; en este sentido, la función formativa requiere un proceso de evaluación democrático, no solamente es responsabilidad del personal docente, requiere de un diálogo continuo con el estudiantado.

Lo anterior establece un debate epistemológico de la evaluación de los aprendizajes en la actualidad, debido a que, desde la corriente naturalista o constructivista, ecológica y crítica, parte del hecho de que la evaluación pretende mejorar los procesos de aprendizaje del estudiantado. Por medio de la mediación pedagógica, contextualiza la realidad del estudiantado, en la adquisición de competencias que le permitan 
desempeñarse en cualquier situación de su vida, por lo tanto, es fundamental partir de los conocimientos previos del estudiantado, contextualizados en su realidad.

Según Díaz y Hernández (2002), desde el constructivismo, el personal docente es un mediador entre conocimiento y aprendizaje del estudiantado, por tanto, en la mediación pedagógica, se comparten experiencias y saberes en un proceso de negociación o coconstrucción del conocimiento. También, según Ausubel (1987), se promueven aprendizajes significativos, que tengan sentido y sean funcionales para el estudiantado. Conjuntamente, se da énfasis en los procesos de construcción de los aprendizajes y en la organización de este mismo.

Para Good y Brophy (1999), se encuentra una gran diversidad de aportes del constructivismo, al aprendizaje y a la evaluación: el concepto de red de estructuración del conocimiento; el conocimiento como construcción social y la zona de desarrollo próximo; el aprendizaje situado y las tareas auténticas en la construcción social del conocimiento; el andamiaje y la transferencia de responsabilidad para el aprendizaje; por tanto, existen diversas corrientes epistemológicas que brindan aportes desde el constructivismo, entre las cuales tenemos:

- La "teoría del esquema" tiene su origen en los trabajos de Ausubel (1998) y Rumelhart, (1980), quienes plantean que en las personas, los conocimientos, habilidades y destrezas se originan en esquemas cognitivos, así como se fundamentan en el principio del aprendizaje significativo, es decir, cuando la nueva información es asimilada mediante un esquema cognitivo previo, por consiguiente, cuantas más representaciones de experiencias de diversa índole se le presenten al estudiantado acerca de determinado tema, se le facilita establecer puentes entre lo que ya sabe y lo nuevo que se le representa.

- La "teoría ecológica o socio cognitiva", según Chauveau, (1992, citado por Condemarín y Medina, 2000), considera fundamental tomar en cuenta el contexto donde se construye el aprendizaje, por tanto, es interaccionista; cuando la persona aprende, intervienen los procesos sociales junto con los cognitivos. Desde la misma perspectiva, el aprendizaje se establece como un puente de aspectos cognitivos y del contexto donde se construye.

- El “enfoque etnográfico", el cual considera que existen ciertos fenómenos del conocimiento humano que se conocen exclusivamente observándolos en su interacción con el contexto, desde una visión holística de la realidad.

- La práctica "pedagógica reflexiva" surge desde los trabajos de Dewey (1989), a principios del siglo XX y Zeichner (1993, citado por Tallaferro, 2005), donde la persona docente, mediante un acto metacognitivo, comprende desde la totalidad los procesos áulicos, con el propósito de mejorarlos y crear el saber pedagógico. Según Díaz y Hernández (2002), el personal docente es un mediador reflexivo que piensa críticamente su práctica, toma decisiones y soluciona problemas pertinentes al contexto de su mediación pedagógica.

Desde el punto de vista de la evaluación de los aprendizajes, el aporte de las anteriores corrientes constructivistas es muy amplio. Entre otros aspectos, se centra en visualizar los procesos más que los resultados, también, se enfatiza en democratizar los procesos evaluativos.

Por lo anterior, la evaluación de los aprendizajes se representa mediante un diálogo de las personas participantes, desde diversos contextos donde interviene la investigación acción; además, privan las técnicas etnográficas como la observación, el estudio de casos y las entrevistas.

Por su parte, deja de tener un carácter eminentemente instrumental y se convierte en un saber ético, donde se considera la diversidad del estudiantado en la eliminación de todo tipo de barreras para el aprendizaje y la participación.

De acuerdo con Salinas (2013), el aprendizaje no puede ser relegado a la enseñanza, es decir, solo basta esta para que el estudiantado aprenda, especialmente contenidos, objetivos, habilidades o competencias, cuyo 
planteamiento se consigna en los diversos programas de estudio. De lo que se trata es no separar el proceso de aprendizaje de la evaluación, y de dar la oportunidad al personal docente para la utilización de técnicas y estrategias muy diversas para tomar en cuenta los distintos estilos y ritmos de aprendizaje de la población estudiantil.

Por tanto, la evaluación de los aprendizajes es un proceso en el cual se emiten juicios de valor sobre las actuaciones y producciones del estudiantado, mediante la construcción y comunicación para la toma de decisiones y la mejora de la educación; de donde se derivan diversas finalidades, evaluación diagnóstica, formativa y sumativa.

Para emitir estos juicios, se basa en las evidencias recolectadas por medio de diferentes modalidades de evaluación y la aplicación de técnicas e instrumentos contextualizados en la realidad donde se genera el conocimiento. Las modalidades de evaluación según la persona que evalúa incluyen: la que realiza la persona sobre sí misma (autoevaluación), la que realizan otras personas (heteroevaluación) como el profesorado, personal tutor o facilitador, así como entre compañeros o compañeras (evaluación entre iguales o coevaluación). Estos tipos de evaluación se deben aplicar de forma complementaria y colaborativa para el beneficio de todas las personas involucradas desde la función formativa de la evaluación de los aprendizajes.

\section{La función formativa de la evaluación de los aprendizajes}

El propósito formativo de la evaluación es precisamente valorar el desempeño individual, colaborativo (el estudiantado se auto organiza) y cooperativo (el estudiantado es organizado por el personal docente) del estudiantado, realimentar y proalimentar directamente el proceso; además obtener información para la toma de decisiones, según las necesidades de cada estudiante.

Existe una serie de preguntas generadoras que se refieren a la utilización de la función formativa en la mediación pedagógica:

- ¿Cuáles instrumentos permiten un registro detallado acerca del desarrollo del trabajo individual y en subgrupos, que se lleva a cabo en el trabajo escolar cotidiano?

- ¿Qué cambios metodológicos son necesarios para aumentar la relevancia del aprendizaje durante la mediación pedagógica?

- ¿Se utilizan estrategias cooperativas o colaborativas para atender el estudiantado con necesidades educativas especiales, altas capacidades, privación económica, discapacidad, migrante entre otros?

Durante la función formativa de la evaluación de los aprendizajes, se puede recopilar información al observar la valoración del desempeño del estudiantado, se aprovechan diferentes tipos de escalas e instrumentos, así como otras técnicas de recogida de información. Algunos de estos instrumentos y técnicas pueden ser: la observación, la entrevista, el registro de desempeño, las rúbricas, el portafolio, el mapa semántico y otros que el personal docente considere apropiados, como las estrategias cooperativas.

Para que la función formativa cumpla su propósito de realimentación - proalimentación permanente, debe de ser ágil y simple, para lo cual se deben aplicar diversas estrategias didácticas y técnicas con la finalidad de que el registro de información permita la toma de decisiones inmediata, para motivar y aumentar el interés de sus estudiantes; contextualizar el proceso de aprendizaje al medio, proponer cambios metodológicos y recursos didácticos cada vez que sean necesarios.

\section{La función formativa de la evaluación de los aprendizajes}

La función formativa de la evaluación de los aprendizajes requiere de los procesos de realimentaciónproalimentación para ir evaluando los objetivos, contenidos o competencias del estudiantado en la mediación 
pedagógica; desde una visión compleja utiliza los recursos propios de su asignatura y los del entorno a la hora que evalúa al estudiantado. Además, prepara desde un sentido de proalimentación, para enfrentar a la población estudiantil para retos similares en un futuro próximo; busca en el estudiantado enfrentar los problemas cotidianos, por medio de diversos recursos, tanto, en el salón de clases como en su cotidianeidad.

De acuerdo con la función formativa, "propone la posibilidad de aplicar estrategias diferentes en las pruebas objetivas, de tal manera que facilitan la valoración de los aprendizajes significativos, el progreso individual de los alumnos y la evaluación continua de los procesos del aprendizaje" (Dolores, Corella y Membreno. 2008, p. 71).

Lo anterior permite al estudiantado tener claridad acerca de la resolución de casos, de problemas, ejercicios, evidencias de desempeño o competencias que se le evalúan por medio de los indicadores y criterios de evaluación durante la función formativa; en este sentido, el proceso de evaluación de los aprendizajes va de la mano de la mediación pedagógica, es decir, tanto el estudiantado como el personal docente utilizan técnicas y estrategias didácticas para ir recopilando información que permite a este último la toma de decisiones inmediata para realimentar - proalimentar.

El propósito formativo de la evaluación de los aprendizajes se convierte en parte inherente al proceso de aprendizaje, mediante los procesos de construcción y comunicación de los significados que se desenvuelven dentro del salón de clases de forma natural. Además, el personal docente debe invitar al estudiantado a la búsqueda y análisis de información que le hace reconocerse como una persona crítica y reflexiva de su propio proceso de aprendizaje.

Por lo tanto, la realimentación durante el proceso de aprendizaje facilita, a la población estudiantil, autoevaluarse y autocorregirse, pero también procesos de transferencia de conocimiento a nuevos contextos, lo que requiere del diálogo y negociación de los participantes en el proceso de aprendizaje, junto con el de evaluación, la cual va configurando los procesos de enseñanza y aprendizaje.

\section{Los procesos de la realimentación - proalimentación}

De acuerdo con Rodríguez e Ibarra (2011), "la evaluación sostenible es un proceso continuo cuya finalidad es que el estudiante sea capaz de autorregular su aprendizaje. En el camino hacia la autorregulación, podemos utilizar estrategias como la realimentación y proalimentación” (p. 6). La característica principal de la autorregulación es que el estudiantado regula su propio proceso de aprendizaje y decide cómo evaluarlo.

Por tanto, la evaluación debe ir de la mano con el proceso de aprendizaje, según Rodríguez e Ibarra (2013), desde el constructivismo, la cual se fundamenta en tres principios:

Las tareas de evaluación deben promover el tipo de aprendizaje necesario para el lugar de trabajo en el contexto sociocultural del siglo XXI.

Los procesos de evaluación deben implicar a los estudiantes de forma activa, promoviendo las competencias que van a necesitar como "aprendices a lo largo de la vida", su capacidad evaluadora para tomar decisiones y orientar su propio proceso de aprendizaje.

Se realiza una retroalimentación prospectiva o proalimentación (feedback as feedforward), es decir, se ofrecen orientaciones y recomendaciones a los estudiantes que sean factibles de realización en un futuro inmediato pudiendo modificar/mejorar su trabajo y nivel de desempeño. (p. 446)

Aunado a lo anterior, la realimentación es abierta, flexible y compartida en cuanto al conocimiento; por esto, promueve y maximiza las oportunidades del aprendizaje, lo cual no la reduce a sus resultados sino a sus procesos. Estos procesos de aprendizaje son estratégicos, se plantean para la vida, es decir, se aprende en un contexto especifico transferible a otros escenarios profesionales y vocacionales. Además, la población estudiantil pone énfasis en tener un papel activo y autónomo en el aprendizaje, el cual le sirva en la toma 
de decisiones, la autoconciencia de las necesidades formativas y la autorregulación del propio proceso de aprendizaje.

Para Rodríguez e Ibarra (2012), la retroalimentación [realimentación] es “información útil y relevante facilitada al estudiante sobre su proceso, nivel competencial y resultados a través de la cual puede mejorar su desempeño. Esta información puede ser facilitada por el profesorado, los compañeros o por el propio estudiante" (p. 4).

Por lo anterior, es importante considerar que, durante la realimentación, el cuerpo docente brinda al estudiantado información útil para la mejora del desempeño presente, por lo que el error se vuelve una fuente valiosa que le permite la autoevaluación y autorregulación de los propios procesos de aprendizaje.

Asimismo, la realimentación es necesaria en los casos en que se pretenda lograr un aprendizaje significativo, o se quiera mostrar al estudiantado el logro de un mayor avance en las distintas áreas, ya sea en la parte académica, vocacional o incluso en su cotidianeidad; esto, con el fin de avanzar con sus objetivos, contenidos o competencias y alcanzar logros deseados, para así poder participar en la sociedad de una manera segura y proactiva.

Por otra parte, según Rodríguez e Ibarra (2012), la proalimentación es:

...información útil, relevante y prospectiva facilitada al estudiante sobre su proceso, nivel competencial y resultados a través de la cual, además de mejorar su desempeño concurre la posibilidad de modificar o mejorar en un futuro inmediato. Esta información puede ser facilitada por el profesorado, los compañeros o por el propio estudiante. (p. 4)

Es decir, brinda la oportunidad, a cada estudiante, de reflexionar acerca de las evidencias del desempeño; permite la generalización a futuras tareas académicas o laborales. Por lo tanto, es un requisito clave desde el propósito formativo de la evaluación, es decir, se encuentra orientado hacia el aprendizaje.

Según Cano (2016), “el feedback [realimentación] puede ser un importante catalizador para la mejora. Para dar apoyo al desarrollo continuado del trabajo en curso, es fundamental proveer al estudiante con aportaciones útiles que le permitan ver los problemas desde nuevas perspectivas" (p. 14). También, promueve la creación de un espacio en donde el estudiantado se favorece del aprendizaje a lo largo de toda la vida.

Por su parte, la proalimentación es necesaria porque es la herramienta perfecta para tomar en cuenta el error, una oportunidad de reflexión del estudiantado acerca de sus procesos de enseñanza y aprendizaje, sumamente importantes, cuando se trabaja con poblaciones vulnerables, donde se medían con una serie de apoyos de todo tipo (físicos, tecnológicos curriculares y otros).

Asimismo, ambos procesos de la realimentación - proalimentación permiten al personal docente y al estudiantado, durante la mediación pedagógicas, una serie de evidencias del avance estudiantil con el propósito de tener una idea clara del aprendizaje significativo de este. De acuerdo con Cano (2016):

El feedforward [pro alimentación] es el proceso que sugiere utilizar la retroalimentación para la mejora del desempeño o del aprendizaje en ocasiones futuras. Se trata de que cada persona se cuestione dónde va, cómo está yendo y hacia dónde debe ir para que el feedback [realimentación] se convierta en feedforward. (p. 34)

Según lo anterior, el personal docente, por medio de la proalimentación, media el aprendizaje, a través de la valoración del desempeño, el cual acerca los aprendizajes a la evaluación, cuando la población estudiantil se desenvuelve en contextos similares a la vida diaria, de esa manera se demuestra lo que ha aprendido por medio de productos concretos, resolviendo un problema, simulando un banco, una pulpería o supermercado y otros.

En síntesis, dentro de los principales aportes de los procesos de realimentación - proalimentación, en la mediación pedagógica permite construir y confirmar el aprendizaje que se produce durante todo el proceso; se convierten en procesos oportunos, debido a que el personal docente valora el logro de las competencias, contenidos y objetivos desarrollados en clase y la capacidad de aplicarlos en nuevos contextos y situaciones futuras. También, brinda la oportunidad a la población estudiantil para reflexionar críticamente, acerca de sus esfuerzos y de su desempeño, por medio de la autoevaluación, la coevaluación y la evaluación entre iguales. 
Por consiguiente, le da la oportunidad al personal docente para que valore su propia práctica profesional y construya una visión de proalimentación con la población estudiantil.

Con base en lo anterior, desde la pedagogía crítica y constructivista, el personal docente tiene la oportunidad de promoverlos durante su mediación pedagógica, por lo que se vuelve fundamental la calificación en el componente de trabajo cotidiano, es decir, según el Reglamento de Evaluación de los Aprendizajes vigente, todas aquellas actividades educativas que el estudiantado realiza con la supervisión docente, trabajo que se observa de forma permanente como parte de un proceso y no de un producto.

\section{Trabajo escolar cotidiano}

El trabajo escolar cotidiano se centra en la mediación pedagógica, donde precisamente el proceso de realimentación - proalimentación se convierte en un diálogo, entre el personal docente y estudiantado, sobre el desempeño del estudiantado en el aula. Ello conlleva un proceso de reflexión-acción del estudiantado, donde se evidencian las fortalezas y se toma conciencia de las debilidades, en busca de la mejora continua de su aprendizaje.

De acuerdo con Soto (2001), "el trabajo cotidiano, por lo tanto, se convierte en una labor que favorece la obtención de información no solo con respecto al desempeño del estudiante en el aula, sino también información que le permita al maestro realimentar su práctica docente, aspectos que beneficiarán las futuras experiencias en las aulas" (p. 13).

Los antecedentes en Costa Rica se remontan al Instructivo técnico administrativo de evaluación III Ciclo y Educación Diversificada (1995), donde se "conceptúa el trabajo cotidiano, las experiencias de aprendizaje, cuyo propósito para el estudiantado es aprovecharlas y utilizarlas para la adquisición de conocimientos y destrezas o mejorar el nivel de alguna habilidad. Se evalúa en forma continua durante el desarrollo de las lecciones como parte del proceso de aprendizaje y no como producto" (MEP. 1995, p. 89).

La calificación del trabajo cotidiano es el resultado de la información recopilada a través de guías de observación tales como listas de cotejo, escalas de calificación y otras que cada docente construya para tales fines.

De acuerdo con Castillo y Cabrerizo (2003), el trabajo cotidiano es la actividad realizada en el centro educativo por el estudiantado, “actividad central y nuclear del proceso de enseñanza y aprendizaje” (p. 244).

En los últimos años, la conceptualización y el propósito del Reglamento de Evaluación de los Aprendizajes, vigente, no ha cambiado sustancialmente, ya que no se concibe la calificación del trabajo cotidiano en forma focal, sino que el personal docente debe calificar integralmente a cada estudiante, según sus particularidades y la presencia en las lecciones.

De acuerdo con lo precedente, la calificación del trabajo cotidiano se visualiza únicamente en términos de logro o no logro, la realimentación queda supeditada para informar fortalezas y debilidades del estudiantado, es decir, en la justificación de una calificación, debido que el personal docente debe informar periódicamente al padre, madre o persona encargada.

De acuerdo con el Ministerio de Educación Pública (2015), para calificar el trabajo cotidiano, se requiere:

Durante el desarrollo de las actividades de mediación, el docente utiliza instrumentos: escalas de calificación, registros anecdóticos, registros de desempeño, rúbricas, listas de control, entre otros, que les permiten, recopilar información (cuantitativa y/o cualitativa) válida y confiable, acerca el desempeño de los estudiantes (p. 1).

La función docente es valorar el desempeño del estudiantado para realimentar, de forma pronta y oportuna, los procesos de enseñanza y aprendizaje. Además, el número de observaciones que se realice queda supeditado al desempeño de cada estudiante y debe ser sistematizado en algún instrumento con indicadores del logro de conocimientos, actitudes, habilidades y destrezas. 
El problema que se presenta es dejar de lado, en la calificación del trabajo escolar cotidiano, los procesos de autoevaluación, coevaluación y evaluación entre iguales.

\section{La autoevaluación, coevaluación y la evaluación entre iguales}

Cuando se mencionan procesos de autoevaluación, coevaluación y evaluación entre iguales, es precisamente la persona quien realiza la evaluación. Se entiende por autoevaluación cuando es la misma persona quien se evalúa, durante los procesos de enseñanza y aprendizaje, por medio de una autorreflexión crítica. Rodríguez e Ibarra (2013) señalan que, en la autoevaluación, el estudiantado logra juzgar su propio desempeño y productos en pro y contra, participa en la emisión de juicios de su propio aprendizaje y es responsable de su propio aprendizaje, es decir, logra la reflexión crítica de su propio progreso y propio aprendizaje.

$\mathrm{Al}$ ser la autoevaluación una metacognición acerca del propio progreso del estudiantado, requiere que desde edad preescolar se vaya creando una cultura evaluadora, para crear una madurez que permita, plenamente, juzgar los propios logros y errores.

Por su parte, la coevaluación, de acuerdo con Rodríguez e Ibarra (2012), es la "modalidad participativa de evaluación que implica un proceso mediante el cual los docentes, junto con los estudiantes, realizan un análisis y valoración de forma colaborativa, conjunta y consensuada sobre las actuaciones, producciones y/ o productos de aprendizaje" (p. 1).

Es importante indicar que en la coevaluación van a estar inherentes los procesos de realimentación y de proalimentación, con la participación tanto del estudiantado como del personal docente.

La evaluación entre iguales es, precisamente, cuando la población estudiantil participa en una valoración sobre su desempeño o actuación, ya sea individual, de toda la clase o en un subgrupo de trabajo; también se evidencian los procesos de realimentación y de proalimentación. Por tanto, "esta posición es mantenida por Roberts (2006), quien define el término 'entre iguales' evaluación se refiere al proceso de reflexión crítica en el que se ven implicados los estudiantes" (Rodríguez e Ibarra, 2012, p. 200). Para llevar a cabo la función formativa de la evaluación de los aprendizajes, en el componente del trabajo cotidiano, es importante que el personal docente promueva medios evaluativos, los cuales consisten en la utilización de todas las estrategias didácticas, técnicas e instrumentos evaluativos que tienen el propósito de promover los procesos de aprendizaje, para responder a la diversidad, al mismo tiempo minimizan todo tipo de barreras desde las políticas, culturales y prácticas del centro educativo.

Una vez que se ha conceptualizado, desde la pedagogía crítica y desde el constructivismo, la función formativa de la evaluación para evaluar el componente del trabajo escolar cotidiano, a continuación, se presenta la ruta metodológica de la investigación.

\section{Ruta METOdológica}

De acuerdo con Guardián (2007), el presente estudio se basa en la investigación cualitativa- interpretativa. Este tipo de investigación la llevan a cabo las personas participantes en situaciones sociales para mejorar sus propias prácticas, el entendimiento de estas mismas y las situaciones dentro de las cuales tienen lugar. Este tipo de investigación se lleva a cabo a partir de una serie de fases.

Con el propósito de conocer cómo califican el componente de trabajo cotidiano, se realiza la investigación con 21 docentes de III Ciclo de la Educación General Básica y la Educación Diversificada, actualmente laboran en la Educación Diurna del Ministerio de Educación Pública. Por su parte, la opinión del investigador trata de comprender a las personas y los procesos de calificación con la función formativa de la evaluación de los aprendizajes, sin trazar visiones preconcebidas, la persona investigadora considera todas las perspectivas como valiosas, y la mediación pedagógica donde se evalúa el trabajo cotidiano es digna del estudio. 
Las personas que participan en el estudio son 21 docentes con nombramiento en propiedad o en interinazgo del III ciclo de la Educación General Básica y Educación Diversificada de las Direcciones Regionales de Educación de San José Norte, San José Central, Cartago, Heredia, San Carlos y Coto, durante el mes de junio del año 2014. Fueron observadas impartiendo lecciones de 80 minutos, durante cuatro ocasiones y luego se realizaron preguntas acerca de la forma de calificar el componente de la evaluación el trabajo cotidiano. Además, se contó con la colaboración de estudiantes de II año de carrera de la Universidad de Costa Rica, en las asignaturas objeto de la investigación: Matemáticas, Español, Música, Estudios Sociales, Psicología, Filosofía, Ciencias y Francés. Además, se observó a una docente de la asignatura de Vida Cotidiana o Educación para el Hogar.

El estudio presenta un muestreo teórico, según Gurdián (2007), lo que se descubre acerca del componente de trabajo cotidiano contrasta con la teoría y los procesos de paradigma crítico.

\section{Categorías de análisis}

En la Tabla 1 se presentan las categorías y subcategorías de análisis, su conceptualización y su operacionalización.

TABLA 1

Categoría de análisis

\begin{tabular}{|c|c|c|c|}
\hline Categorias & Definición & Subcategorías & Operacionalización \\
\hline $\begin{array}{l}\text { Trabajo } \\
\text { cotidiano }\end{array}$ & $\begin{array}{l}\text { Se entiende por } \\
\text { trabajo cotidiano } \\
\text { todas las } \\
\text { actividades } \\
\text { educativas que } \\
\text { realiza el } \\
\text { alumnado con la } \\
\text { guía de su docente. } \\
\text { Este trabajo se } \\
\text { observa en forma } \\
\text { continua, durante } \\
\text { el desarrollo de las } \\
\text { lecciones, como } \\
\text { parte del proceso } \\
\text { de aprendizaje y } \\
\text { no como producto. } \\
\text { Para su calificación } \\
\text { se debe utilizar la } \\
\text { información } \\
\text { recopilada con las } \\
\text { escalas de } \\
\text { calificación y otros } \\
\text { instrumentos } \\
\text { técnicamente } \\
\text { elaborados. } \\
\text { (Artículo } 23 \\
\text { Reglamento de } \\
\text { Evaluación de los } \\
\text { Aprendizajes (MEP, } \\
\text { 20o9). }\end{array}$ & $\begin{array}{l}\text { Realimentación-proalimentación } \\
\text { La autoevaluación, } \\
\text { coevaluación, autorregulación y } \\
\text { la evaluación entre iguales }\end{array}$ & $\begin{array}{l}\text { Se realiza un análisis de } \\
\text { contenido de la mediación } \\
\text { pedagógica, con el fin de } \\
\text { interpretar críticamente la } \\
\text { pertinencia de las } \\
\text { estrategias utilizadas para } \\
\text { la calificación del trabajo } \\
\text { cotidiano con la función } \\
\text { formativa de la evaluación } \\
\text { de los aprendizajes. Se } \\
\text { refiere a los agentes } \\
\text { evaluativos que el personal } \\
\text { docente utiliza con el } \\
\text { propósito de que el } \\
\text { estudiantado se pueda } \\
\text { evaluar a símismo, evalué } \\
\text { a sus compañeros jas, a la } \\
\text { vez que es evaluado. } \\
\text { También, que tenga la } \\
\text { potestad de decidir qué } \\
\text { desea aprender, lo cual } \\
\text { requiere de su preparación } \\
\text { para que enfrente sus } \\
\text { propios procesos de } \\
\text { aprendizaje en cualquier } \\
\text { momento o lugar. De } \\
\text { acuerdo con Rodriguez e } \\
\text { Ibarra (2011), la evaluación } \\
\text { entre iguales es cuando el } \\
\text { estudiantado, de forma } \\
\text { critica y reflexiva, evalúa } \\
\text { los aprendizajes de sus } \\
\text { compañeros jas. }\end{array}$ \\
\hline
\end{tabular}

Nota: Elaboración propia. 


\section{A. Descripción de los instrumentos}

\section{Observación}

Por medio de la observación no participante, quien investiga y las personas colaboradoras no se involucran con el contexto, con el propósito de registrar las experiencias acerca de la calificación del trabajo de primer orden. Según Gurdián (2007), proporciona una descripción no participante de las personas observadoras. El rol principal de la persona investigadora, según Gurdián (2007), "es recoger datos y el grupo estudiado es consciente de las actividades de observación del investigador” (p. 196). Se confeccionan una guía de observación para definir la primera categoría de análisis que se presenta al inicio de este marco metodológico.

\section{Entrevistas}

Según Gurdián (2007), la entrevista cualitativa se caracteriza porque no tiene forma directiva, sino que es más bien abierta, semiestructurada, no estandarizada. Tiene, como propósito principal, alcanzar un enfoque conversacional, y no necesariamente de preguntas y respuestas. A su vez, Gurdián (2007) explica que la entrevista debe ir estructurada por una "Guía de tópicos", la cual especifique un listado pequeño de temas para conversar de ellos con la persona entrevistada. Esto puede lograrse de treinta a cincuenta minutos de conversación. Esta guía de tópicos tiene que manifestar relación teórica con los objetivos que se quieren alcanzar. Por último, Gurdián (2007) distingue dos tipos de técnicas de investigación dentro de la entrevista cualitativa o abierta: La entrevista en profundidad y la entrevista enfocada.

La primera es holística y tiene como temática "la vida, las experiencias, las ideas, los valores y la estructura simbólica de la o el [sic] sujeto, aquí y ahora" (Gurdián, 2007, p. 200). La segunda se anticipa de acuerdo con un tema de interés, la conversación se dirige hacia ese tema, además la persona seleccionada se elige de acuerdo con este. En el caso de la entrevista acerca del trabajo escolar cotidiano, interesa saber la conceptualización para compararla con el Reglamento de Evaluación de los Aprendizajes vigente, así como conocer, si dentro del trabajo escolar cotidiano, como parte de la función formativa, se llevan a cabo procesos de realimentaciónproalimentación, autorregulación del aprendizaje y la participación del propio estudiantado en el proceso evaluativo.

\section{Discusión de los RESUltados}

Se analizan los resultados con base en una interpretación crítica propia del investigador y personas colaboradoras de investigación: Lugares, características de procedencia de la población meta, instancias refieren, procesos se efectúan para el análisis del trabajo cotidiano con la función formativa de la evaluación de los aprendizajes. Además, se plasma un proceso de triangulación que determina la fiabilidad y la credibilidad de la información, por medio de la utilización de las técnicas de entrevista y la observación no participante.

\section{Proceso de realimentación -proalimentación}

A continuación se presenta la conceptualización que tiene el personal docente del trabajo cotidiano, asimismo, durante la función formativa de la evaluación, los procesos de la realimentación - proalimentación; la autoevaluación, coevaluación, autorregulación, evaluación sustentable y la evaluación entre iguales.

La función formativa de la evaluación de los aprendizajes contribuye al desarrollo personal del estudiantado. También, facilita información que permite conocer el estado de aquellos indicadores 
cognitivos, conductuales, procedimentales y afectivos en cada estudiante. En opinión de una docente de III ciclo de la Educación General Básica:

\begin{abstract}
La importancia de la función formativa en el proceso educativo es que permite la aplicación de diferentes estrategias para observar en cuáles aspectos, el estudiantado ha mejorado, cuáles temas ha comprendido mejor, en cuáles no ha asimilado lo requerido. Dichos aspectos son importantes en este tipo de evaluación, debido a que las estrategias de cooperación son poco utilizadas en nuestros centros educativos, principalmente en secundaria, en donde cada quien se las ingenia para hacer su trabajo, sin tomar en cuenta a su compañero/a. Nuestra preocupación es que el estudiantado no genere escándalo y desorden en el momento de trabajar de forma cooperativa. (Comunicación personal con docente de la asignaturas básicas y complementarias de la Educación Diversificada, martes 9 de setiembre del 2014)
\end{abstract}

De lo anterior, se infiere que, en la calificación del trabajo cotidiano por parte del personal docente, lo realiza por medio de la técnica de observación, utilizando una gran variedad de estrategias para ir realimentando los procesos de enseñanza y aprendizajes; sin embargo, le es difícil aplicar estrategias de aprendizaje cooperativo, con el fin de no causar ruido, por lo que se promueve un aprendizaje más individualista.

Por tanto, la evaluación formativa pretende basarse en las fortalezas propias de cada estudiante para luego realizar un proceso cooperativo/colaborativo, pues este tipo de evaluación es diferenciador de lo que conocemos con la aplicación de instrumentos de medición, ya que se centra en todas las áreas y utiliza el "error" como otra forma de abordar el aprendizaje.

Cuando se va a observar al personal docente en su mediación pedagógica, luego se le pregunta, cómo realiza la evaluación del trabajo cotidiano, lo que se encuentra es ejemplos como el siguiente:

El docente observado pertenece al área de Ciencias Naturales, por un periodo de dos lecciones, este se encontraba dando clases de Física a un grupo de décimo año de aproximadamente 27 personas. La clase se desarrolló básicamente de la siguiente forma: el profesor, con ayuda de un proyector y una presentación en Power Point, estaba estudiando las tres leyes Newton, él procedía a explicar lo que venía en la presentación y les decía que era lo que tenían que escribir. Luego se procedió a realizar una práctica, que después resolvió el estudiantado y la revisaron en la pizarra con ayuda del profesor, esto con el fin de aclarar dudas. Posteriormente se le preguntó cómo califica el trabajo cotidiano. La respuesta fue la siguiente:

Bueno el mayor porcentaje lo asigno a las diferentes prácticas que realizamos en clase, ya que después de cada práctica les reviso y al final del trimestre reviso sus cuadernos para verificar que tengan todo lo que se ha realizado en clase. Sin embargo, cuando realizamos diferentes actividades utilizo algunas tablas de observación para evaluar su desempeño. (Comunicación personal con docente participante, 2014)

De acuerdo con el párrafo anterior, el personal docente califica el trabajo cotidiano por medio de prácticas sumativas, por cumplir un requisito utiliza los instrumentos de observación en el desempeño del estudiantado; sin embargo, no logra explicar el cómo las realiza. Por tanto, para la aplicación de la función formativa durante los procesos de enseñanza y aprendizaje, a la hora de planificar las estrategias didácticas que se van a ir desarrollando durante la mediación pedagógica, se necesita plantear las siguientes interrogantes: ¿Tienen claros los conceptos el estudiantado?, ¿es adecuada la forma de presentar el tema?; así como tomar en cuenta también el intercambio entre pares y entre docentes y estudiantes acerca de la evaluación. De ahí la importancia de las actividades de repaso antes de iniciar un tema o un contenido nuevo, así como tener un registro de la información en relación con las actitudes y el comportamiento de la población estudiantil cuando se elabora cualquier tipo de actividad. La observación se vuelve una herramienta muy útil, pues permite objetivar y explicitar los criterios con los que se valora el desempeño del estudiantado, aunque al conocer lo estipulado en el Reglamento de Evaluación de los Aprendizajes vigente, se realizan ciertas modificaciones a conveniencia.

El docente de Estudios Sociales y Educación Cívica, que imparte clases en los niveles sétimo y décimo año, por medio de una plática indicó que él se basa en los componentes de evaluación que ha estipulado el Reglamento de Evaluación de los Aprendizajes (MEP, 2009), sin embargo, intenta ver de una manera más 
humana esto y suele emitir su criterio por medio de lo que él observa del educando, principalmente esfuerzo, interés y actitud, esto también lo refleja en el componente de concepto, que en palabras del propio docente:

Si lo hiciera de manera totalmente matemática ellos son los que salen perdiendo

Este docente menciona que a la hora de poner la calificación de este componente

...suele redondear hacia arriba y tenderles la mano a los que se lo merecen.

Lo anterior presupone, necesariamente, un error por parte del docente; al confundir la calificación del trabajo cotidiano con el componente del concepto, lo atribuye a su buena voluntad para no perjudicar al estudiantado, por tanto, el personal docente con la realimentación lo que hace es generalmente revisar ejercicios o problemas en la pizarra, para vincularlos con la propia realidad del estudiantado y que este indique cómo sería un producto de calidad. Sin embargo, aún hace falta ese vínculo de la proalimentación con el propio contexto del estudiantado, que proporcione ejemplos de la calidad que espera el estudiantado de lo que es un buen desempeño. Por tanto, Salinas (2013) considera que desde un carácter meramente instrumental a un carácter eminentemente ético:

Los profesores actuamos, con respecto a la evaluación, no sólo en estrecha dependencia con la forma como nos relacionamos con la disciplina que enseñamos, sus campos de producción y el saber sobre la enseñanza, la creación de ambientes de aprendizaje, la construcción de proyectos, la lectura de los diferentes contextos, sino también con las concepciones éticas y políticas que configuran nuestra propia historia. Así mismo, los estudiantes se construyen para si una concepción, que deviene fundamentalmente de la forma como han sido evaluados. De esta manera, la evaluación se puede convertir en un aliado para el seguimiento y la transformación, o en artificio que se aprende, para burlar la obtención de resultados (pp., 4-5).

Lo anterior conlleva considerar la evaluación de los aprendizajes desde su atributo ético, debido a las formas de evaluar al estudiantado por parte del personal docente, así actuarán en su propia vivencia cotidiana. Para que el personal docente logre en el salón de clases los procesos de proalimentación y la autorregulación tendría que llegarse a democratizar la función formativa de la evaluación de los aprendizajes, los procesos de autoevaluación, coevaluación y la evaluación entre iguales, desde procesos de autorregulación, para que la población estudiantil tenga una participación dinámica en los procesos de aprendizajes que van de la mano con los procesos evaluativos.

Por tanto, los procesos de evaluación de los aprendizajes se podrían democratizar en la mediación pedagógica, mediante la autoevaluación, coevaluación y evaluación entre iguales; para trascender la cultura de evaluación de producto en el trabajo cotidiano, al estudiantado, cuando aprende, se le debe dar la oportunidad de expresarse conscientemente de múltiples maneras en la mediación pedagógica, la construcción de un mapa, portafolio, de una exposición, un debate, un video, pues a la vez que son formas de expresar su aprendizaje, también son medios evaluativos que permiten evaluar un proceso de adquisición del conocimiento.

\section{La autoevaluación coevaluación, autorregulación y la evaluación entre iguales}

Como se indicó arriba, las modalidades de evaluación según la persona que evalúa incluyen: la que realiza la persona sobre sí misma (autoevaluación), la que realizan otras personas (heteroevaluación) como el personal docente, de tutoría o facilitador, así como entre compañeros y compañeras (evaluación entre iguales o coevaluación). Estos tipos de evaluación se deben aplicar de forma complementaria y colaborativa para el beneficio de la población estudiantil.

De acuerdo con Vallejo y Molina (2014), es necesario retomar la función pedagógica, en cuanto la evaluación no se puede desligar de la mediación pedagógica, por tanto, su fin principal es mejorar la adquisición de los aprendizajes del estudiantado aplicando la autoevaluación, coevaluación y evaluación entre iguales. 
De las observaciones realizadas en diferentes centros educativos, se desprende que existen intentos de docentes por llevar a la práctica la función formativa de la evaluación de los aprendizajes, mediante la autoevaluación, coevaluación y evaluación entre iguales. A continuación se presenta un ejemplo donde se realiza un laboratorio para integrar la teoría con la práctica, con este tipo de estrategias didácticas se observa al estudiantado muy motivado con este tipo de habilidades que se van desarrollando, pese a las limitaciones que hay en dicho colegio. Lo que hace la diferencia, respecto de los deberes docentes según el Reglamento de Evaluación de los Aprendizajes, es el compromiso de dicha profesora para poder integrar estas situaciones a la mediación pedagógica, aunque antes del laboratorio que realizó fue sumamente magistral su lección.

Lo anterior, es predecible, con base en Salinas (2013), en el sentido de que la población estudiantil desde las propias normas reglamentarias del sistema educativo, participe activamente.

[La participación estudiantil], en los procesos de evaluación, incide en el desarrollo de competencias, potencia estrategias de pensamiento reflexivas, críticas e independientes, amplía la capacidad de formular y resolver problemas mejorando las opciones, potencia la capacidad de discusión y negociación, motiva el pensamiento e incrementa el aprendizaje y la confianza. No obstante, participar requiere condiciones de posibilidad, no puede limitarse a la convocatoria de voces sin una plataforma que aporte los elementos necesarios para expresarse con argumentos, con firmeza, con convicción. (p. 9)

Consecuentemente, se interpreta que para lograr procesos democráticos de la evaluación de los aprendizajes, se requiere la plena participación de la población estudiantil mediante la autoevaluación, la evaluación entre iguales y coevaluación, lo cual permitirá un desarrollo estudiantil en habilidades mentales superiores como la resolución de problemas, el pensamiento reflexivo - crítico; además, la capacidad de diálogo y negociación, competencias necesarias en el siglo de las transformaciones tecnológicas. A continuación, se describe el proceso de observación mencionado.

\section{La descripción del contexto de la observación}

El salón de clases es bastante grande, uno de los problemas es que se comparte debido a que hay dos grupos recibiendo clases. La clase inicia sin contratiempo, no se toma lista, la profesora se percata de la ausencia de una estudiante, tomó apuntes, sin mucha demora realiza una introducción de la lección sobre lo que estuvieron realizando la clase pasada, una exposición y se abre como un pequeño espacio para preguntas. La población estudiantil participa bastante. Luego la profesora hace una pequeña introducción sobre la fotosíntesis, al parecer ya han estado viendo el tema en clase, en la lección observada se explicó el ciclo bioquímico de Calvin (proceso de fijación del CO2), un tema complejo y que genera bastantes consultas. La clase se mantenía en silencio prestando atención, pero el estudiantado sentado más atrás se distraía bastante, había mucho ruido que se generaba por el otro grupo con el que compartían el salón, les costaba prestar atención sobre el tema que estaban viendo. La clase finalizó con normalidad, en las lecciones observadas se vio participación de estudiantes, principalmente al inicio, la clase fue muy magistral.

Cuando se entrevista a la docente comentaba que las clases de ella son bastante variadas, le es de gran interés que los estudiantes realmente estén alcanzando los objetivos planteados. Con respecto a la calificación del trabajo cotidiano, se observó:

Las evaluaciones cotidianas las hace semanalmente de acuerdo con las observaciones que realiza, además de la participación estudiantil, cuando nota que el estudiantado no tiene interés en el tema apunta lo que observa, si alguien tiene más de tres apuntes en el mes habla con él o ella para saber que está sucediendo, cuando hace clases magistrales o practicas se trata de integrar el uso del celular y conexión a internet para la obtención de información o imágenes.

Como se puede deducir, la docente se preocupa por que sus estudiantes se integren a las actividades que realiza en la mediación pedagógica, cuando encuentra desmotivación por parte del estudiantado, establece un proceso de diálogo, además busca, con el uso de las tecnologías, establecer nuevas formas de participación y trabajo cooperativo tan necesario para las estrategias cooperativas en el salón de clases. Barberá(2006) explica, 
en relación con los aportes que tiene la tecnología a la e-Evaluación, que por medio de ella se promueven tres proceso de aprendizaje: la evaluación automática, referida a la validación de las respuestas de manera inmediata; la enciclopédica, por medio del acceso a la información, por internet, y la colaborativa, utiliza medios de construcción grupal como foros, wikis. Por tanto, desde los medios digitales, la evaluación aún va más unida a los procesos de aprendizaje.

Debido a ello, en la anterior observación, a pesar de las limitaciones que presenta el centro educativo, la profesora organiza laboratorios de acuerdo con los temas que lo ameriten Explica que por trimestre hace aproximadamente tres laboratorios. Sumando este trabajo al cotidiano, para estos laboratorios, sus estudiantes tienen una libreta, en ella explica los objetivos de la práctica, materiales que se utilizarán y el procedimiento a seguir. Ya para el trabajo de laboratorio, sus estudiantes tienen que mantener el orden del improvisado laboratorio, el manejo de los reactivos (alcohol, hielo, yodo, formol) y en la libreta manejan cuadros para las observaciones de dichos laboratorios. Para la siguiente semana, el estudiantado, por medio de una exposición, tiene que dar una explicación científica de dicho laboratorio; esto como manera de reporte de laboratorio, con ello hace un nexo entre lo teórico y lo práctico.

Todos estos trabajos tienen un porcentaje dentro del trabajo cotidiano, para cada uno de los tres pasos de la rubricas evaluativas (Libreta, trabajo de laboratorio y exposición). Al final de los laboratorios, el grupo de trabajo entrega a la profesora una autoevaluación y una evaluación entre iguales y la autoevaluación que también es tomada en cuenta.

Con base en lo anterior, la función formativa del trabajo escolar cotidiano se logra cuando el estudiantado se involucra más, no solamente en los aprendizajes, sino también con la evaluación, tal y como lo señala Boud (2010), la docente prepara a la población estudiantil para que sean capaces de enfrentar exitosamente su proceso de aprendizaje a lo largo de la vida.

Sin embargo, existen docentes que no tienen claridad sobre cuáles son los indicadores que se deben ir evaluando en el trabajo escolar cotidiano, al no lograr los procesos de autoevaluación, coevaluación y la evaluación entre iguales, no logran la función formativa de la evaluación de los aprendizajes, los procesos de proalimentación y la autorregulación. Por ejemplo:

Hoy por hoy evalúo según el estudiante trabaje en el aula, aplicando las normas y conocimientos que uno le va dando, como el orden y aseo, respeto, originalidad -que no se copien-, la capacidad de no solo trabajar de forma individual, y de estar atento con la materia que uno explica. Yo ahí también les tomo puntillos de presentación personal, al final de cuentas de eso se trata lo que enseño. Yo hago mi propia tabla, una hoja por cada estudiante y los voy llamando uno a uno dependiendo como me acomode, para revisarle el cuaderno, así les doy un poco de chance para no afectarlos cuando faltan.

De acuerdo con el párrafo anterior, la docente entrevistada evalúa el trabajo escolar cotidiano, sin tener claridad de lo que indica el Reglamento de Evaluación vigente, ni los demás lineamientos emanados por parte de las autoridades magisteriales. Lo más lamentable es que reduce todo el proceso de evaluación a la simple revisión del cuaderno, como se infiere en la observación, califica aspectos muy difíciles de observar como la originalidad, creatividad, orden y aseo, por lo que demuestra dejar de lado el desempeño propio de la asignatura.

Es importante tomar en consideración que cada asignatura tiene sus propias demandas y oportunidades para poder evaluar el trabajo escolar cotidiano, por ejemplo:

Siendo el rubro más alto el de trabajo cotidiano él explica que se le es demasiado complicado crear rúbricas o listas de cotejo, etc. Pues, tiene muchos grupos y muy poco tiempo. Por lo que en trabajo cotidiano se les evalúa básicamente que cumplan con los materiales que deben llevar a la clase (flauta y cuaderno de pentagrama). Y su método no es sumativo, sino, que se parte del hecho de que todos tiene 100 de nota. Y que conforme falten con materiales o no terminen los trabajos en clase (sellos), se les va restando puntaje de la nota de cotidiano.

De la anterior, la observación demuestra que la función formativa de la evaluación del trabajo escolar cotidiano se reduce a que el estudiantado aporte los materiales, deja de lado los procesos de enseñanza 
y aprendizaje, que se vienen desarrollando en la mediación pedagógica, de acuerdo con las habilidades o competencias del programa de estudios.

Aunado a lo anterior, cuando se entrevista a una docente de la asignatura Español, que trabaja en III Ciclos de la Educación General Básica, nos indica algo muy parecido a lo precedente:

Depende del nivel por ejemplo en sétimo año es $25 \%$ hay una serie de rubros que se toman en cuenta, los rubros como no dicen a ciencia cierta, los hemos establecidos los profesores como por ejemplo si el estudiante trae material a clase, que si el estudiante trabaja, que si el estudiante copia o no copia el trabajo; etc diferentes rubros. Pero para todos en octavo año es $15 \%$ y en noveno año es $15 \%$.

En la precedente observación se demuestra que la función formativa de la evaluación de los aprendizajes, en el trabajo escolar cotidiano, se establece desde un punto de vista instrumental, donde no se visualiza el proceso de observación, ni los procesos de realimentación o pro alimentación, únicamente aporte de materiales o lo que copia en el cuaderno el estudiantado.

Otra docente de la asignatura Francés, del III Ciclo de la Educación General Básica y Diversificada, se refiere a su forma de calificar el trabajo cotidiano:

Es un trabajo el cual desarrolla gradualmente y clases a clase, utiliza el espejo de clase para un poco agilidad en tomar las notas por la cantidad de estudiantes que tienen sus grupos, normalmente de 35 en adelante, checa uno por uno el aporte de materiales y durante la clase se acerca a los estudiantes a aclarar dudas y ver que estos avancen con lo que hagan. En las prácticas orales las realiza tanto de manera grupal como individual, poco a poco saca el tiempo para esto, a forma individual realiza menos que grupal por el factor tiempo, pero a nivel grupal las realiza constantemente para desarrollar la pronunciación de la mejor manera.

En la anterior observación, la docente trasciende de calificar únicamente el aporte de materiales y realiza procesos de realimentación grupal y trabajo cooperativo.

Las anteriores entrevistas evidencian que desde la función formativa de la evaluación de los aprendizajes, el componente del trabajo cotidiano se ve reducido a aspectos, "aporte los materiales", "realizar los ejercicios del cuaderno" y "copiar de la pizarra"; por tanto, se convierte dicha calificación en un acto conductual de otorgar sellos, o porcentajes, dejando de lado los procesos de proalimentación, la sostenibilidad y la participación estudiantil de una forma crítica y reflexiva, de manera que le permita utilizar su propio aprendizaje en nuevos contextos.

Ello conllevaría a avanzar, de acuerdo con Monereo (2009), hacia los principales postulados de la evaluación auténtica, donde el personal docente es el encargado de mediar y recrear los ambientes de aprendizaje, propiciando procesos de proalimentación, o actuaciones futuras. El proceso evaluativo va ligado al de aprendizaje, donde la población estudiantil demuestre sus habilidades en una diversidad de contexto de la vida diaria.

Por consiguiente, para poder evaluar el trabajo escolar cotidiano es necesario tomar en cuenta el contexto donde se lleva a cabo la mediación pedagógica y espacios directamente relacionados con las propias vivencias del estudiantado.

De lo anterior se deduce que, de acuerdo con las observaciones realizadas, se omite la función formativa de la evaluación de los aprendizajes, en la calificación del trabajo escolar cotidiano, debido a que, en la mayoría de los casos, el personal docente la emplea de una forma muy tradicional, como por ejemplo, de acuerdo con las observaciones realizadas: revisado el cuaderno por medio de sellos, el aporte de materiales, si copia de la pizarra, o tratando de observar del estudiantado el interés, creatividad o participación en el salón de clases, lo anterior, demuestra que no hay claridad conceptual de la calificación de dicho componente. 


\section{RefLeXiones Finales}

Dentro de las principales conclusiones en la calificación del trabajo escolar cotidiano, se encuentra que el personal docente observado y posteriormente entrevistado aún no logra tener claridad sobre la forma en que se califica el componente, como parte de la función formativa de la evaluación de los aprendizajes. Una de las consideraciones develadas es, precisamente, que se requiere un diálogo permanente en el contexto del aula, donde se utilicen, a parte de la observación, técnicas más participativas como la entrevista, estudio de casos, portafolios, mapas, con el fin de obtener mayores evidencias del desempeño del estudiantado.

Con el propósito de democratizar la evaluación de los aprendizajes, es fundamental, asesorar al personal docente en los procesos de realimentación, proalimentación, evaluación sostenible y autorregulación del aprendizaje, es decir, el aporte de las corrientes epistemológicas constructivistas y de la pedagogía crítica a la evaluación formativa; por tanto, el trabajo escolar cotidiano es, ante todo, la mediación pedagógica reflexiva, contextualizada al conocimiento previo del estudiantado, desde una realidad compleja y diversa, donde el error es garante de seguir aprendiendo.

Por lo anterior, se parte de la función formativa de la evaluación de los aprendizajes, en un sentido de la realimentación - proalimentación, lo cual permite al personal docente reflexionar, en un momento dado, acerca del proceso para verificar en qué medida se han alcanzado, por parte del estudiantado, las competencias, contenidos u objetivos y, en función de la información obtenida, proceder a programar actividades que se relacionen con la propia realidad.

El estudiantado debe tener un papel protagónico en la evaluación del trabajo escolar cotidiano, lo cual le permite aplicar las técnicas e instrumentos de evaluación, antes de enfrentarse a un adecuado desempeño, por lo que las actividades de aprendizaje que realiza el personal docente en su mediación pedagógica deben relacionarse estrechamente con la evaluación de los aprendizajes, tanto en el contexto, conocimientos previos, aplicabilidad en la vida cotidiana, como en la transferencia de estos.

Las expectativas del desempeño muchas veces varían entre lo que considera el cuerpo docente y lo que realmente puede resolver el propio estudiantado, por lo tanto, son fundamentales los procesos de autoevaluación, coevaluación y evaluación entre iguales.

Resulta primordial la conformación de equipos de trabajo con el personal docente, técnico administrativo y la comunidad educativa, con el fin de llevar a cabo procesos de democratización de la evaluación, como es la autoevaluación, coevaluación y evaluación entre iguales, para lo cual la evaluación de los aprendizajes no debe ser una responsabilidad exclusiva del personal docente.

Por su parte, en la mediación pedagógica, la promoción de estrategias didácticas cooperativas y colaborativas, en la atención a la diversidad para la plena participación del estudiantado, sin ninguna barrera para el aprendizaje, le permite autoevaluarse y coevaluarse, aspectos esenciales en la función formativa de la evaluación.

Se requiere una práctica docente reflexiva, mediante el trabajo compartido entre el personal docente con otros profesionales de psicología, trabajo social, educación especial, psicopedagogía, entre otros, para formular apoyos pedagógicos al estudiantado con discapacidad, talentoso, migrante y privado económicamente.

Para la calificación del trabajo escolar cotidiano, se requiere conocimiento y comprensión del significado de la mediación adaptada al currículo con "apoyos pedagógicos" concretos, en la atención de las fortalezas individuales para satisfacer la diversidad en el salón de clases. También, a la aplicación de la función formativa de la evaluación de los aprendizajes con las estrategias de evaluación con los aportes del constructivismo, donde se visualice la evaluación desde el punto de vista ético, más que desde una función instrumentalista.

Ante una calificación conductual del trabajo escolar cotidiano, se muestra una clara evidencia de que la mediación pedagógica sigue siendo tradicional. 


\section{REFERENCIAS}

Ausubel, D. (1998). Psicología educativa: Un punto de vista cognoscitivo. México: Mc Graw - Hill.

Barberá, E. (2006) Aportaciones de la tecnología a la e-Evaluación. Revista de Educación a Distancia, 6(1), 1-13.

Boud, D. (2010). Siete propuestas para la reforma de la evaluación en la educación superior. Sydney: Australian Learning and Teaching Council.

Cano, E. (2016). Del feedback al feedforward. En N. Cabrera y M. R. Mayordomo (Eds.), Feedback formativo en la universidad. (pp.31-40). Barcelona: Colección Transmedia XXI.

Casanova, M. (1999). Manual de evaluación educativa. Madrid: Editorial La Muralla.

Castillo, S. y Cabrerizo, D. (2003). Evaluación educativa y promoción escolar. Madrid: Pearson Educación.

Condemarín, M y Medina, A. (2000). La evaluación auténtica de los aprendizajes. Santiago, Chile: Andrés Bello.

Dewey, J. (1989). ¿Cómo pensamos? Cognición y desarrollo humano. Barcelona, España: Paidós.

Díaz, F. y Hernández, G. (2002). Estrategias docentes para un aprendizaje significativo. Una interpretación constructivista. México: McGraw-Hill.

Dolores, H., Corella, M. y Membreno, J. (2008). Evaluación de los aprendizajes. San José: CECC.AECID.

Good, T. y Brophy, J. (1999). Psicológica educativa contemporánea. México: Mc Graw - Hill.

Gurdián, A. (2007). El paradigma cualitativo en la investigación socio educativa. San José: CECC-IDER.

Ministerio de Educación Pública. (1995). El instructivo técnico administrativo de evaluación de I, II, III Ciclos de la Educación General Básica y la Educación Diversificada. San José: Departamento de Evaluación Educativa.

Ministerio de Educación Pública. (2009). Reglamento de evaluación de los aprendizajes. San José: Departamento de Evaluación de los Aprendizajes.

Ministerio de Educación Pública. (2015). Respuestas a las consultas más frecuentes del proceso evaluación de los aprendizajes. San José: Departamento de Evaluación de los Aprendizajes.

Monereo, C. (2009). La autenticidad de la evaluación. En M. Castelló (Coord.), La evaluación auténtica en enseñanza secundaria y universitaria. (pp. 9-22). Barcelona: Edebé, Innova universitas. Recuperado de https:// docs.wixstatic.com/ugd/a03747_8e05e7a5694b478987d6ced11071957e.pdf

Roberts, T. (2006). Self, Peer and Group Assesment in E-Learning. Hershey, PA: Information Science Publishing. doi: https://doi.org/10.4018/978-1-59140-965-6

Rodríguez, G. y Ibarra, M. (2011). De la calificación a la e-proalimentación. Estrategias y herramientas innovadoras para la evaluación/proalimentación y el desarrollo de competencias en los estudiantes universitarios. Cadiz: Universidad de Cádiz, Evalfor.

Rodríguez, G. y Ibarra, M. (2012). e-Evaluación orientada al e-Aprendizaje estratégico en educación superior. España: NARCEA.

Rodríguez, G. y Ibarra, M. (2013). Autoevaluación, evaluación entre iguales y coevaluación: conceptualización y práctica en las universidades españolas. Revista de Investigación en Educación, 11(2), 198-210. Recuperado de http://reined.webs.uvigo.es/ojs/index.php/reined/article/view/708

Rumelhart, D. (1980). Schemata: The building blocks of cognition. San Diego: La Jolia Calif, Center for Human Information Processing, University of California.

Salinas, M. (2013). La evaluación como dispositivo para la participación. Colombia. Universidad de Antioquía.

Salinas, M. y Rodríguez, H. (2013). La evaluación educativa y el paradigma crítico. Colombia. Universidad de Antioquía.

Salinas, M. y Rodríguez, H. y Cuervo, B. (2013). La evaluación educativa y el paradigma estructural interpretativo: Cualitativo, hermenéutico o fenomenológico. Colombia. Universidad de Antioquía.

Soto, R. (2001). Evaluación del trabajo cotidiano: Una competencia del docente en el aula. Revista Actualidades Investigativas en Educación 1(2), 1-13. Recuperado de http://revista.inie.ucr.ac.cr/uploads/tx_magazine/ TRABAJO_COTIDIANO.pdf 
Vallejo, M., Molina, J. (2014). La evaluación auténtica de los procesos educativos. Revista Iberoamericana de Educación, 64, 11-25.

Tallaferro, M. (2005). La formación para la práctica reflexiva en las prácticas profesionales docentes. Revista Venezolana de Educación: EDUCERE. 10(33), 269-273. Recuperado de http://www.saber.ula.ve/ bitstream/123456789/20065/2/articulo8.pdf

\section{BY-NC-ND}

